\title{
SSE Path Routing Protocol for WSN IOT Using Fuzzy Mean Weighted Code and Enthalpy ANN-GOA
}

Poonam Rani ( $\sim$ ponam2132@gmail.com )

Maharishi Markandeshwar Engineering College

Avinash Sharma

Maharishi Markandeshwar Engineering College

\section{Research Article}

Keywords: Wireless Sensor Network, cluster, packet code, ANN, GOA.

Posted Date: August 2nd, 2021

DOI: https://doi.org/10.21203/rs.3.rs-596213/v1

License: (c) (1) This work is licensed under a Creative Commons Attribution 4.0 International License. Read Full License 


\title{
SSE Path Routing protocol for WSN IOT Using Fuzzy Mean Weighted Code and Enthalpy ANN-GOA
}

\author{
Poonam Rani ${ }^{*}$, Avinash Sharma ${ }^{2}$ \\ ${ }^{1}$ Research Scholar, Department of Computer Science and Engineering, Maharishi Markandeshwar Deemed \\ University, Mullana, Haryana, India \\ ${ }^{2}$ Professorin theDepartment of Computer Science and Engineering, Maharishi Markandeshwar Deemed \\ University, Mullana, Haryana, India \\ *Email id: yashvidabas@gmail.com
}

\begin{abstract}
:
Wireless Sensor Networks (WSNs) play a key role in the Internet of Things (IoT) by delivering cost-effective solutions for specific IoT applications. The amount of energy used to transport data from a sensor node to its destination is a significant consideration when developing wireless sensor network routing strategies. A variety of routing protocols are utilised in WSN to improve network performance. When a node's mode transitions from active to sleep, the efficiency declines because data packets must wait at the starting point where they were transmitted, which increases packets' waiting time and end-to-end latency, resulting in an increase in energy consumption. This study proposes the SSE Path routing protocol as a Scalable, Secure, and Energy Efficient Routing Protocol for WSN IoT. Our proposed protocol, first selecting the nodes using a single-hop clustering algorithm. Second, FMWC (Fuzzy Mean Weighted Code) based packet coded operation for the security of packet. Finally, Scalable Enthalpy Artificial Neural-Network Based Grasshopper Optimization Algorithm (EANN-GOA) algorithm for selecting the congestion-free path for transit the data packets. The experimental results revelations the dominance of presented protocol comparing with the existing protocols.
\end{abstract}

Keywords: Wireless Sensor Network, cluster, packet code, ANN, GOA.

\section{INTRODUCTION}

Because of recent scientific developments in the fields of microelectronics, sensing machines, and wireless communication systems, wireless sensor networks have attracted worldwide attention (WSNs). WSNs are made up of a variety of low-power devices called motes or sensor nodes. They connect with one another via wireless media and carry out a variety of functions, such as environmental monitoring and battlefield observation, among others. The sensor nodes, on the other hand, face a variety of obstacles, including limited transmission diversity, processing capacity, garage capacity, and power resources [1].WSNs are made up of a large number of battery-powered sensor nodes that convert observed data into electrical signals for subsequent processing. The WSN has no infrastructure, and the sensors are dispersed haphazardly [2]. The WSN's job is to collect data from the network on a regular basis and deliver it to the base station, which then decides what to do nextWSN is crucial in many WSN-assisted IoT applications.[3].

Clustering enables multi-hop transmission, data aggregation, compression, and the removal of redundant data. The benefits of clustering are dependent on the precision of the clustering set of rules and the health of the parameters being used. Unlike distributed clustering algorithms, which employ local information from individual sensor nodes, centralised clustering algorithms provided by the BS provide the most reliable clustering solutions since the WSN's overall view is available [5]. One of the most difficult components of clustering is determining the quality of the clusters. A range of criteria, such as density and separation, can be used to assess cluster quality (inter-cluster and intra-cluster distances). The data in each 
cluster should be close enough together to avoid overlap, and the clusters formed should be unique enough to avoid overlap. The three metrics used to calculate separation are the distance between the closest data in two clusters, the distance between the farthest data in two clusters, and the distance between the centres of two clusters [6].

When it comes to cluster routing, LEACH has been used as a benchmark in the majority of cases. Indeed, LEACH aids in the encapsulation of an energy balancing data-forwarding technique that strives to achieve energy balance. As a result, LEACH has gained widespread adoption due to a goal to extend network longevity while conserving sensor node energy [78].

Clustering is a powerful hierarchical routing-based strategy that splits the network into distinct zones of nodes with similar capabilities, allowing the network to scale more easily. Clustering is a technique for increasing the capacity of a machine by grouping together similar types of sensors. All of the nodes are thought to have similar potential and skills. A node can have a variety of roles assigned to it, including $\mathrm{CH}$, gateway, and cluster member.A $\mathrm{CH}$ is a cluster organiser that facilitates communication within the cluster; on the other hand, a gateway sensor connects sensor signals across clusters and communication ranges [9]. Routing performance is harmed by frequent $\mathrm{CH}$ selection because nodes are preoccupied on node selection rather than communication. The elements that influence cluster formation and $\mathrm{CH}$ communication are still being researched [10-11]. By utilising data aggregation techniques at $\mathrm{CHs}$, the clustering methodology minimises the bulk of acquired data by maintaining only significant information [12].

$\mathrm{CH}$ selection is extremely important in cluster-based routing methods. Most clustering algorithms focus solely on $\mathrm{CH}$ selection and cluster formation [13], rather than data routing after cluster creation. Data about data compactness and separateness can be obtained from clustering outcomes. Furthermore, a large number of clusters can duplicate data on data distributions, which are almost certainly unique from class distributions. Furthermore, the data cannot be analysed based on classification results.As a result, the mixture with clustering outcomes might provide varied but supplemental data for classifications, reducing the uncertainty associated with classification outcomes due to a lack of comprehension or inadequate data [15].

The suggested methodology's key contribution is that it uses the SSE Path routing protocol to pick a congestion-free path for data packet transit from source to destination.

- The clustering technique employs a criterion based on four variables at the next-hop node.

- Protect data packets that are encoded using a fuzzy mean weighted code.

- The Scalable Enthalpy Artificial Neural-Network Based Grasshopper Optimization Algorithm (EANN-GOA) algorithm selects the least congested channel for data packet transmission.

The remainder of the paper is divided into sections as follows: section 2 discusses a review of related work and the issue description, and section 3 describes the SSE path routing protocol in WSN. Then, in part 4, the experimental analyses are described in depth, and lastly, in section 5 , the conclusion is explained.

\section{RELATED WORKS}


Sukhwinder Sharma et.al[16] delivered the Due to actually appropriate absorption of excess energy by advanced nodes, simulation-based overall performance evaluation of the suggested method in comparison to several relevant strategies reveals that HEC produces a longer solid region, higher throughput, and larger electricity dissipation. The stability length development is around 65 percent, 30 percent, 15 percent, and 17 percent for LEACH, SEP, FAIR, and HEC-based SEP protocols, respectively. The scalability of the suggested technique is further investigated using various sector lengths and sensor node counts.

Phet Aimtongkhamet.al [17] deliveredthe basic To deal with traffic congestion induced by temporary events, a congestion management approach was developed. Congestion, on the other hand, is typically caused by a series of anomalies and, as a result, might last for a long period. As a result of this research, a congestion manipulation approach that can handle any sort of congestion has been developed. In three primary areas, the analysis followed a vague set of criteria for addressing traffic congestion: optimal path selection, traffic rate modification using a momentum indicator, and optimal timeout placement for a circuit breaker to prevent continuing congestion.

Muhammad Muzzammil et.al [18] presented the fundamental ideas and concepts of magnetic field communication by defining the major blocks, outlining the issues with each block, and emphasising new advancements The three key blocks that must be recognised are: 1) transmit and acquire coils, 2) matching network, and 3) magnetic field channel characteristics. The first block demonstrates how a single-dimensional coil is utilised to construct a directional communication link between a transmitter and receiver, followed by multi-dimensional and meta-material coils to overcome the directionality problem. The matching network further highlights the distinctions between induction and resonance phenomena, as well as their implications for variety and capacity. The 0.33 block mentions the elements that affect magnetic fields, which are important for underwater wireless communication with magnetic fields. Finally, the research looks at current advancements at the physical, MAC, and network layers.

Quan Wang et.al [19] Firstly, The lemmas for the link between any adjacent layers, the ideal cluster length, and the optimal Cluster Head $(\mathrm{CH})$ distribution, as well as their proofs, were proposed. Furthermore, the 3rd function of Backup Cluster Head $(\mathrm{BCH})$ was described, as well as the accompanying mechanism for rotating jobs among the $\mathrm{CH}$ and $\mathrm{BCH}$, to alleviate the "Hot Spot Problem" and reduce energy consumption caused by the rotation of $\mathrm{CH}$ functions. The protocols for Energy-Efficient Compressive Sensing-based Clustering Routing (EECSR) are then described in detail. Finally, thorough modelling tests were carried out in order to determine its energy efficiency.

Thangaramya et.al [20] presented the intelligent routing in WSNs for IoT is a significant phenomenon that must be addressed if the network's Quality of Service is to be improved (QoS). Additionally, avoiding severe packet loss or packet drop, quick energy depletion, and network unfairness, which results to a reduction in node overall performance and growth input off for packet delivery, the energy required for communication inside IoT-based sensor networks is an important task. As a result, there may be a significant desire to evaluate energy utilisation by utilising nodes to improve overall network performance using software that employs intelligent device learning techniques for making powerful routing decisions.

Poonam Rani et.al [25] provides a linear electronic communication technique that may be scaled up or down according to the application's requirements. The simulation findings show that scaling the network by adding/removing nodes in the same network region has no effect on the network's lifespan, but that if the network's size is increased/decreased, the network's lifetime is improved as well.

Rani Poonam et.al [26] presented a routing scheme is proposed which is predicated on forming a Minimum Spanning Tree (MST) and works on basis of flat based routing technique 
to perfectly utilise vitality and improves the era of the system together with minimising the transmission delay. Transmission between the clusters via Cluster Heads (CH's) has been suggested obsessed on the gap of separation among sensors. Here forms a linear chain among the nodes and connect them in such a fashion that reduces overall cost of the governing system.

Rani Poonam et.al [27] Wireless Sensor Network (WSN) consists of a network with huge quantity of sensors that are deployed to accomplish a selected task. These low cost sensors are proficient of aggregating and communicating the sensed information over the sphere.. However, these nodes being powered by battery are energy constrained. Proposes a routing protocol supported cluster formation among same style of sensor nodes (SN) for gathering of knowledge at intermediate nodes within the cluster and these intermediate nodes further transmits data to resource opulence sink.Many procedures are already to be had withinside the literature on energy-efficient routing for WSN's.

- The existing routing approach reduces the problem of broadcast storm in clustered sensor networks, but it also increases energy consumption and reduces transmission security.

- A scalable and energy-efficient routing system converts long-distance communication into tiny multi-hop distance communication with load balancing, resulting in a lower and more consistent Stability Factor (SF) problem.

\section{PROPOSED SYSTEM}

The deployment strategies, as well as the routing protocol, determine the efficacy of WSN in IoT, particularly large-scale applications. When a network is distributed over a vast area, the performance of WSN-assisted IoT suffers. This piece is a demonstration. SSE Path routing protocol for WSN IoT is a scalable, secure, and energy efficient routing mechanism. Because several sources attempt to deliver their packets to a destination at the same time, which is a common scenario in IoT applications, this protocol is designed to work in networks with high traffic.Our suggested protocol initially employs a criterion that is a function of four factors: residual energy, distance to the sink, node density, and network average energy at the nexthop node in order to pick nodes using a clustering technique. Second, a packet coded procedure based on FMWC (Fuzzy Mean Weighted Code) to ensure packet security. Finally, the Scalable Enthalpy Artificial Neural-Network Based Grasshopper Optimization Algorithm (EANN-GOA) algorithm is used to choose the least congested channel for data packet transmission. The proposed technique is depicted in figure 1 as a block diagram. 


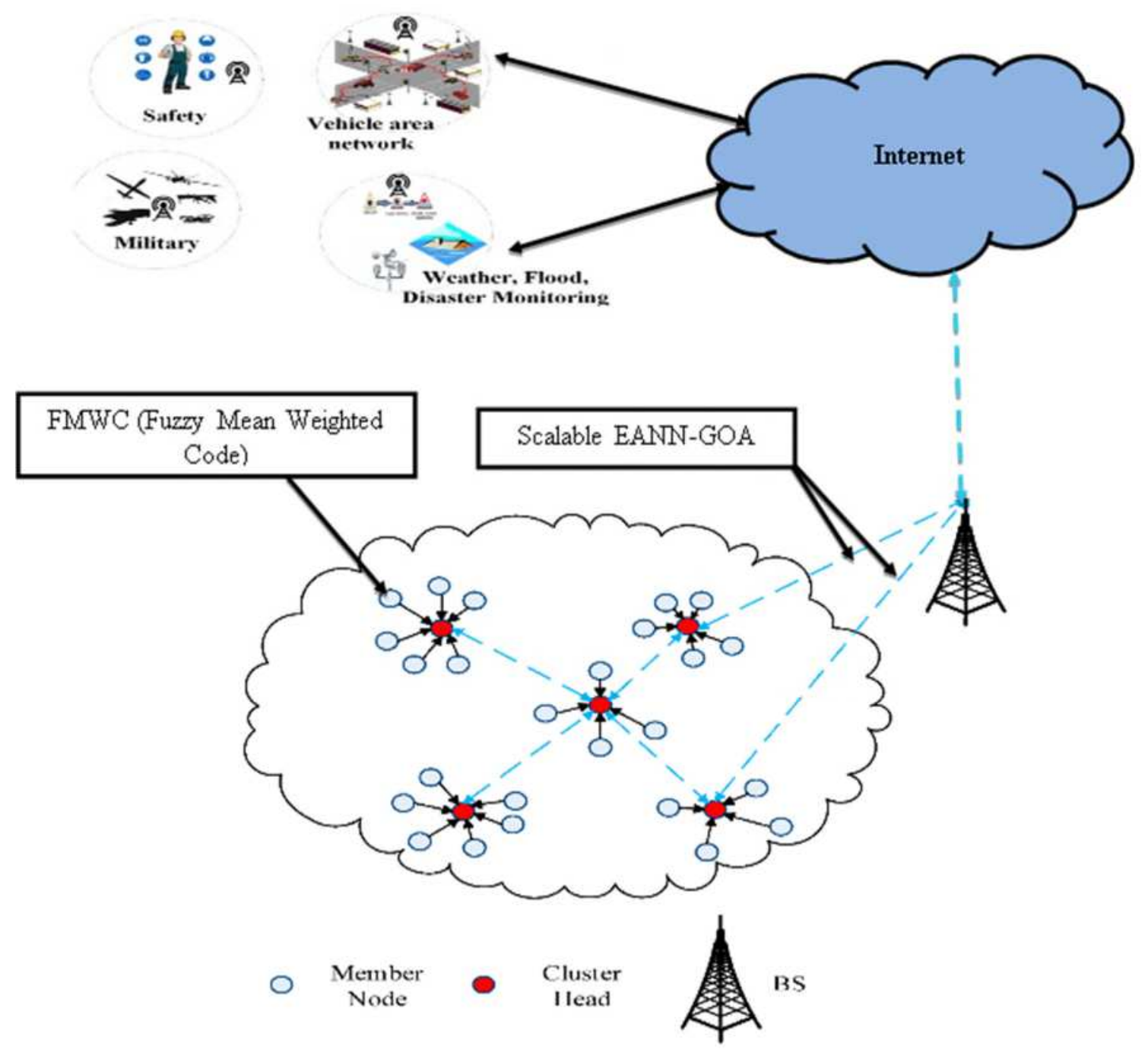

Figure 1: Proposed technique

\subsection{Selecting node using One-hop Clustering}

A WSNs produced of nodes from a few hundred or even thousand, where each node is associated to each other sensorsClustering is a key approach for extending the network period in wireless sensor networks. The routing protocol is a method of determining the best path for data to take from source to destination. The data packets are clustered using the single-hop clustering technique in this case. Single-hop communication occurs in very small sensor networks where the base station and data packets are so close that they can interact directly with one other. Now we'll look at the four factors: Residual Energy, Distance to Sink, Node Density, and Network Average Energy at the next-hop node. The one-hop clustering is represented as follows:Let $C N=\left\{g_{i} ; i=1,2, \ldots . n\right\}$ be cluster nodes, $\mathrm{n}$ be several clusters, $C H=\left\{c_{i} ; i=1,2, \ldots . n\right\}$ be initial centroids, the clustering of the nodes of WSN is shown in figure 2 .

\subsubsection{Residual Energy:}

The energy model depicts the energy level of the network. The node's energy level at the start of the simulation is the initial value of the energy model defined in the node. After routing packets have been received or delivered, the residual energy is the current value of energy in a node. 


$$
R E_{x}=C_{x} * \frac{E_{\text {residual }}}{E_{\text {max }}}
$$

Where $C_{x}$ is the initial percentage of cluster heads among all 'n' nodes, $E_{\text {residual }}$ is represents current residual energy, $E_{\max }$ presents maximum energy.

\subsubsection{Distance to the sink:}

Different distances from the sink generate an imbalance in energy depletion. Based on the distance to the sink and the routing scheme, divide the entire network into a few groups. Each group has a cluster number and a node that belongs to it.

$$
D s_{x}=C * \frac{D_{j}}{\sum_{j=1}^{n} D_{j}}(1 \leq j \leq n)
$$

Here, $D_{j}$ is the distance to the sink and lower index means near to the sink, $C$ represents a cluster in each group.

\subsubsection{Node density}

The node density $\mathrm{N}_{\text {eden }}$ implies the relation tightness among the activity nodes. All nodes would know the number of their neighbors which is called node density. To avoid network congestion, node density can be evaluated and a threshold value for sub-cluster formation can be determined.

$$
N_{d e n}=\sum_{i=1}^{n}\left(\frac{C_{x}}{n}\right)
$$

Whereas $N_{d e n}$ represents node density,n denotes the number of nodes, $C_{x}$ presents cluster in each group.

\subsubsection{Network Average Energy:}

The cluster heads are selected using a probability formula based on the ratio of each node's residual energy to the average energy of the network.

$$
E_{a}=\frac{\sum_{i=1}^{n} E_{\text {residual }}}{n}
$$

Where $E_{a}$ Presents average energy 


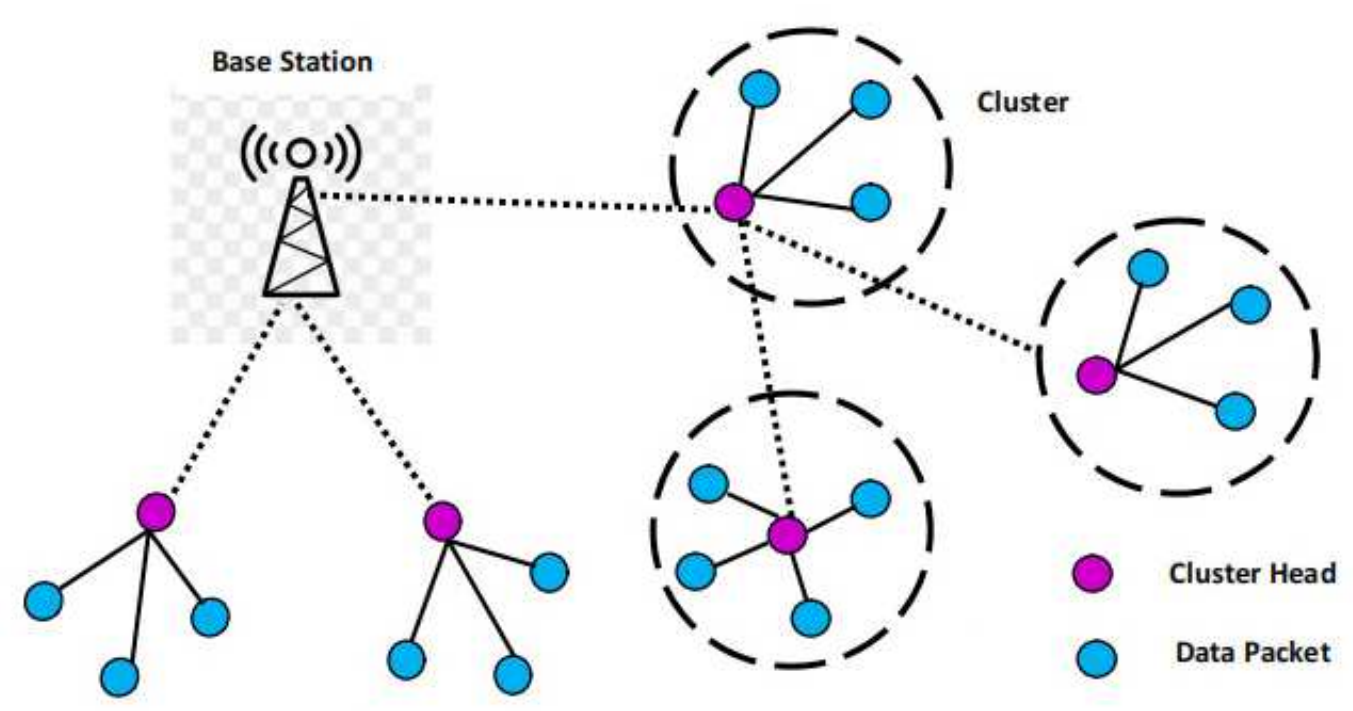

Figure 2: Single-hop clustering Algorithm

\subsection{Packet Coding Method}

The objective of our proposed work is to accomplish a superior usage of the transfer speed and to decrease the network congestion in the multimedia media information dispersal situation. To do as such, the network coding procedure is utilized to decrease the amount of times a message has been retransmitted The source is placed at opposite ends of a comparable street and offers $\mathrm{N}$ hop's space of interest in our proposed study, as shown in Figure 1. Source disseminates non-security information among the vehicles in the common part of the road between them.

Instead of relay broadcasting, our proposed approach uses entropy-based path selection for information transfer and encodes it into a single packet before rebroadcasting it in the network, and in this manner lessens the transfer speed utilization just as postponement at the relay. The relay is thought to have the option to advance the packets and play out an FMWC procedure on the got packets before rebroadcasting them. It is additionally expected that the transfer is mindful of the normal information rates that the source is sending.

\subsubsection{Operational example of TPC}

A basic network topology is explored, with four stations, each of which is within transmission range of the others.A source node $\left(s n_{1}\right)$ sends a data packet $(P k)$ to a destination node $\left(s n_{k}\right)$, which is also sending a packet $(d k)$ to the source station. Furthermore, this special bidirectional communication is supported by two helper or relay nodes $\left(R_{1}\right.$ and $\left.R_{2}\right)$.Pursues is a word that describes the entire procedure.

Direct 1: At direct $T_{1}$, the source node $s n_{1}$ transfers the data packet $P k$ to the destination node $n_{2}$

Direct 2:At direct $T_{2}$, destination node $s n_{2}$ desertions to demodulate the info packet, so propagation a packet request ( $P K R$ ) packet requesting for the cooperation of the neighboring relay nodes along with the data packet $B^{\prime}$, destined to the source node $s n_{1}$. 
Direct 3: The receiving of $P K R$ at $\operatorname{direct} T_{3}$ starts the nodes $R_{1} R_{2}$ and to become started relays and check the trust-based optimized set up their link to participate in the dispute contention phase.

Direct 4: At direct $T_{4}$, the setup link $R_{1}$ terminates and $R_{1}$ transfers the coded packet $P k$ ,$d k$ to the nodes $s n_{1} s n_{k}$ and concurrently.

Direct 5: At direct $T_{5}$, the destination node $s n_{k}$ retrieves the original packet $P k$ and sends back an acknowledgment $(A K E)$ packet subsequently it can decipher legitimately the Adaptive quantum logic-based coded packet.

Direct 6: At direct $T_{6}$, the source node $s n_{1}$ acknowledges the packet $d k$ since it can decode appropriately the coded packet $P k, d k$ the network topology operation with transmitting packet coding is depicted in figure 2.
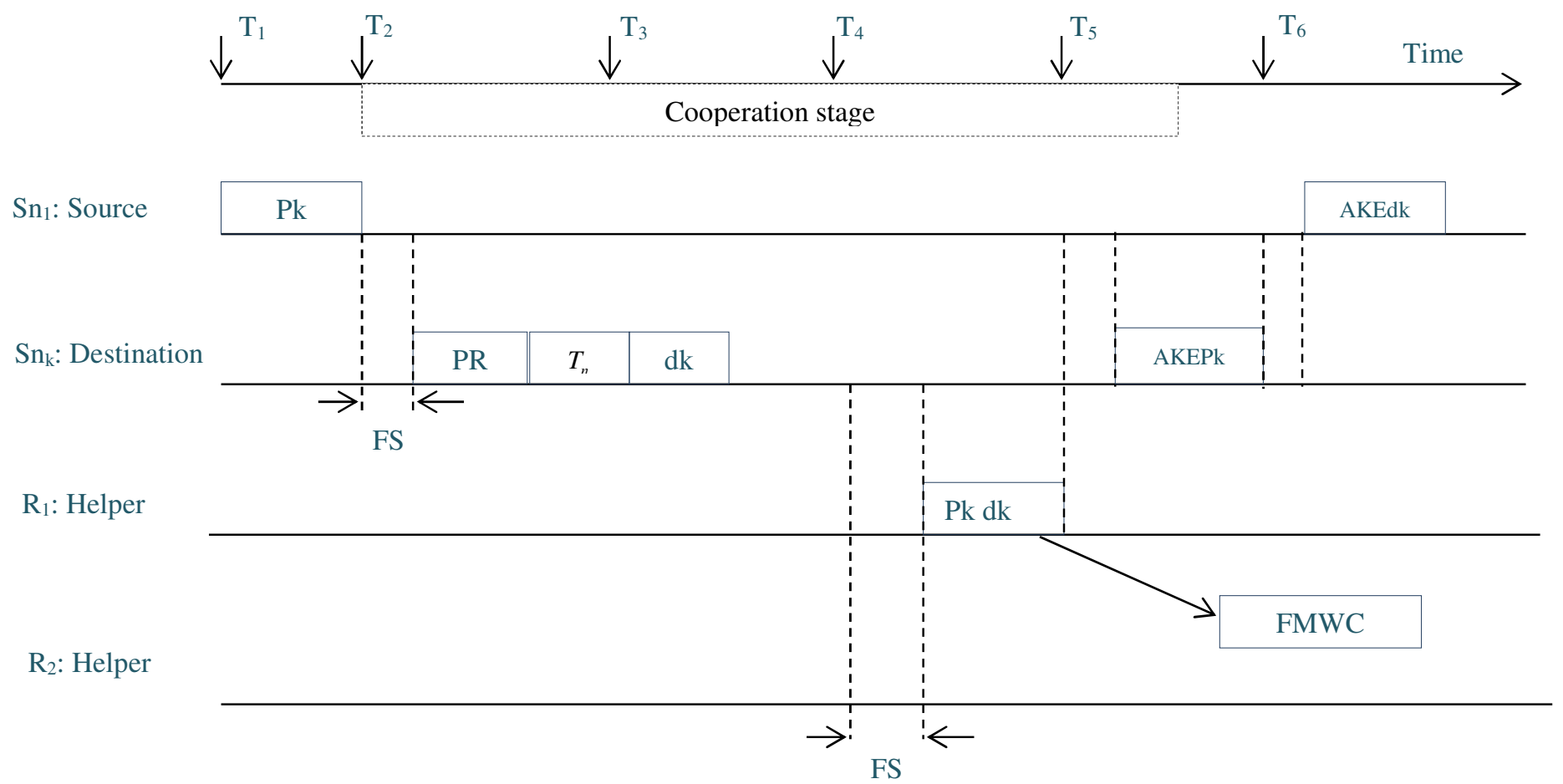

Figure 3: Network topology operation with a frame sequence

\subsection{Fuzzy Mean Weighted Code (FMWC)}

\section{1) Mean weighted value calculation}

The mean weighted value is calculated by multiplying the training data (TD) by the average trust value from section C. (5). Following the calculation of the score value, adaptive fuzzy logic is used.

$$
\mathrm{S}=\text { Mean }\left(t_{1}^{\prime}+t_{2}^{\prime}+t_{3}^{\prime}+t_{4}^{\prime}+t_{5}^{\prime}+t_{6}^{\prime}\right)
$$

Where, $\mathrm{S}$ is the Mean weighted value.

\section{2) Fuzzy membership function}

The process of defining 'crisp' inputs into fuzzy linguistic variables and linking them to membership values is referred to as fuzzy membership performance. The membership 
function indicates the degree to which an element of the comprehensive set is related to the fuzzy set. Within the range of $[0,1]$, a fuzzy set will capture some value. Every score feature (input), as well as the difference in weighted events (input), was categorised into one of three levels: low, moderate, or high. A mathematical representation of membership performance is provided by Equation (6).

$$
A_{\alpha}=\left\{x \in X \mid \mu_{A}(x) \geq \alpha\right\}=\left[\min \left\{x \in X \mid \mu_{A}(x) \geq \alpha\right\}\right] \quad \text { Where, } x=S
$$

\section{3) Fuzzy inference}

For fuzzy inference, a collection of 'If-Then' logic assertions is necessary. A standard fuzzy rule is as follows:

$$
\text { IF } x \text { is } A \text { AND y is BTHEN } z \text {, }
$$

The predecessors are A and B, while the successor is $\mathrm{z}$. The mix of input variables determines the number of fuzzy rules.A pair of fuzzy rules was constructed in total. Table 3 provides an additional example of the principles. In equation, the max-min strategy is linked to fuzzy inferences (7).

\section{4) Defuzzification}

$$
\mu_{A}(x)=\max \left\{\min \left[\mu_{1}(x), \mu_{2}(x), \ldots \ldots \mu_{n}(x)\right]\right\}
$$

The final phase of a fuzzy system is defuzzification. The process entails converting the fuzzy inference results to a crisp number. As a result, the precise output is:

$$
y=\frac{\int \mu(y) y d y}{\int \mu(y) d y}
$$

If $y=$ If the R1 rule is chosen, the outcome will be ' 0 '. The decision will be ' 1 ' if the $=$ Rule id R2 is selected. In this case, the algorithm given;

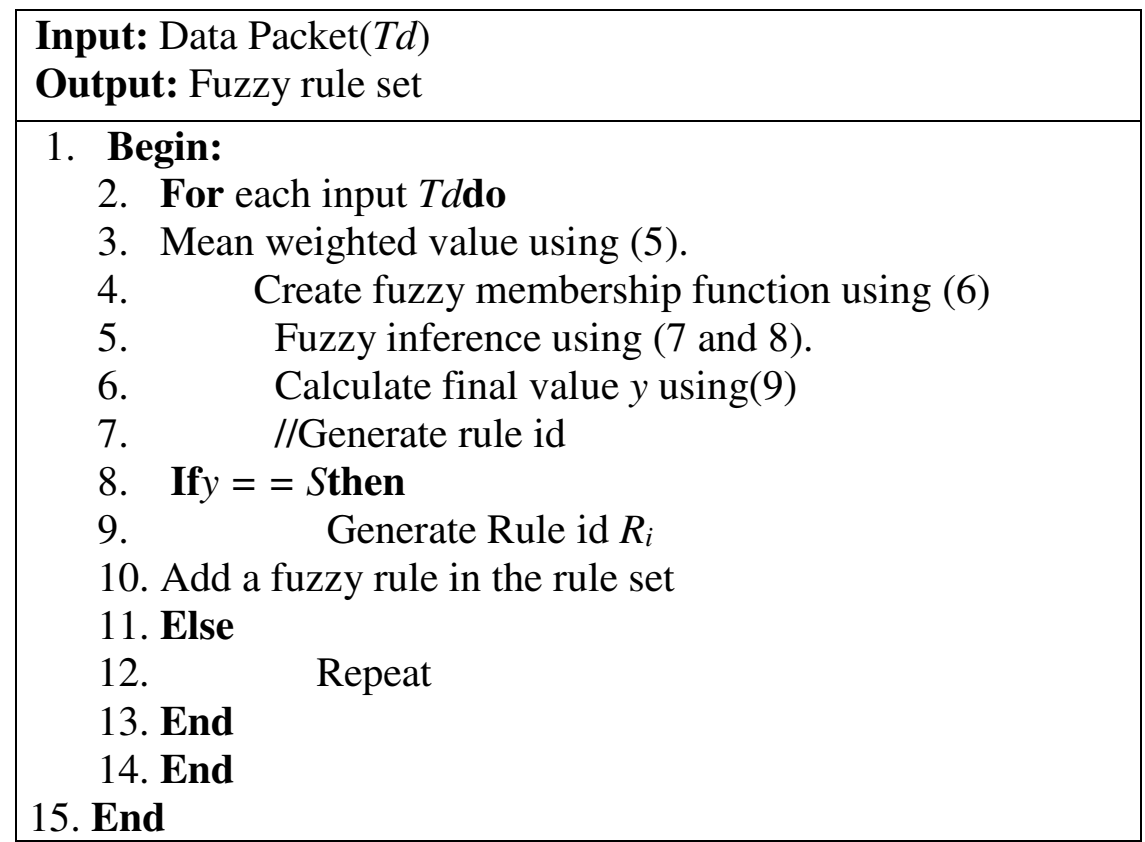

Algorithm: 3 Fuzzy Mean Weighted Code

Finally, the encrypted data packet is securely delivered to the target node through the designated relay nodes. Figure 5 depicts the overall flowchart of the suggested method. 


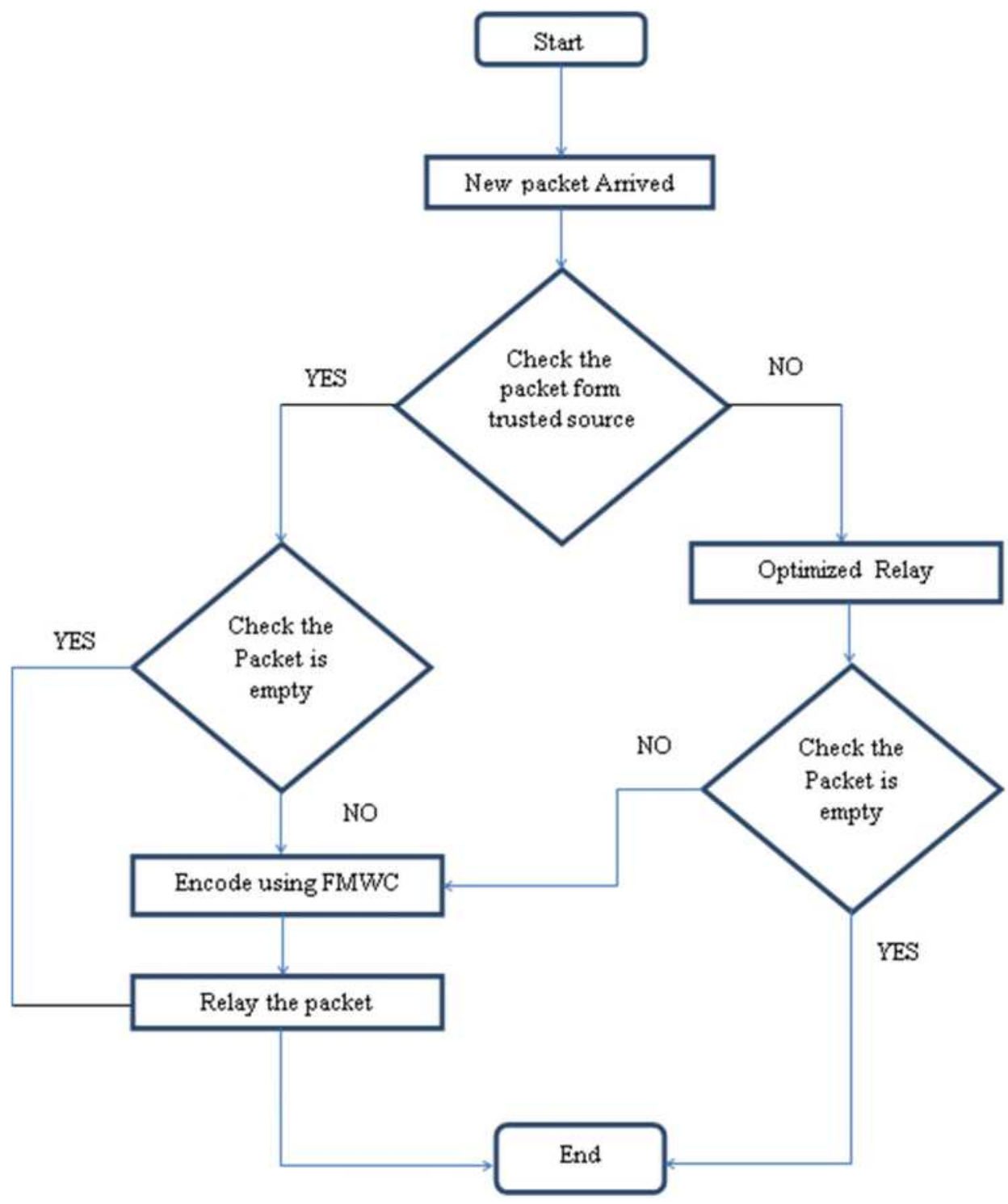

Figure 5: Flow chart of the proposed network coding

\subsection{Scalable Enthalpy Artificial Neural-Network Based Grasshopper Optimization Algorithm (EANN-GOA) algorithm}

Here, the enthalpy is used to select the best routing path. After that encoded the data packets Enthalpy perform the sum of the internal energy and the node pressure and volume of a thermodynamic system which is mathematically represented as follows equation (8),

$$
\mathrm{E}=\mathrm{I}+\mathrm{PV}
$$

Here, $\mathrm{E} \rightarrow$ enthalpy, $\mathrm{I} \rightarrow$ internal energy, $\mathrm{P} \rightarrow$ Network Average Energy, $\mathrm{V} \rightarrow$ Node density.

\subsubsection{ANN-based Grasshopper Optimization Algorithm}

Then, as the input to ANN, use the computed enthalpy values. ANN is used to identify the best path for transferring data packets. In a nonlinear parallel approach called feed forwardback propagation, ANN learns from inputs and goal dataThere are three levels to an ANN: input, hidden layer, and output layer. The number of features in the input data is represented by the nodes in the input layer, while the objective value of the dataset is represented by the nodes in the output layer. An ANN can build a nonlinear mapping between input and output based on the intricate connections between neurons in the hidden layer and the activation 
function in each node. There are three layers in this image: an input layer, one or more hidden layers, and an output layer. Consider a three-layer neural network $(m=3)$ with nl neurons in the lth layer.Taken Straining samples $\left(U_{s}, T_{S}\right), s=1,2, . ., S$, where $U_{s}=\left[u_{1}, \ldots ., u_{n_{1}}^{S}\right]$ and $T_{S}=\left[t_{1}^{s}, \ldots ., t_{n_{M}}^{s}\right]$. For certain inputs $U=\left[u_{1}, \ldots, u_{n_{1}}^{s}\right]$ of the network, the input $\mathrm{x}_{1}^{l}$ and output $\mathrm{y}_{1}^{l}$ of $i$ th neuron of the $l$ th layer.

\section{Input layer}

$$
\left\{\begin{array}{l}
y_{0}^{l}=x_{0}^{l} \equiv 1, \\
y_{j}^{l}=x_{j}^{l} \equiv u_{j}
\end{array} \quad \mathrm{j}=1,2, \ldots, \mathrm{n} l\right.
$$

\section{Hidden layer}

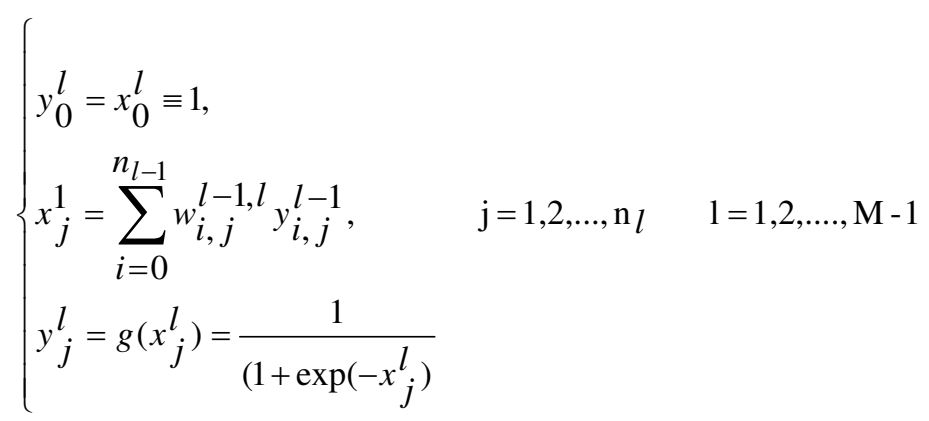

Whereas, $w_{i, j}^{l-1, l}$ is the weight from the $i$ th neuron of $(l-1)$ th layer to the $j$ th neuron of $l$ th layer, and $\mathrm{g}($.$) is the transfer function.$

\section{Output layer}

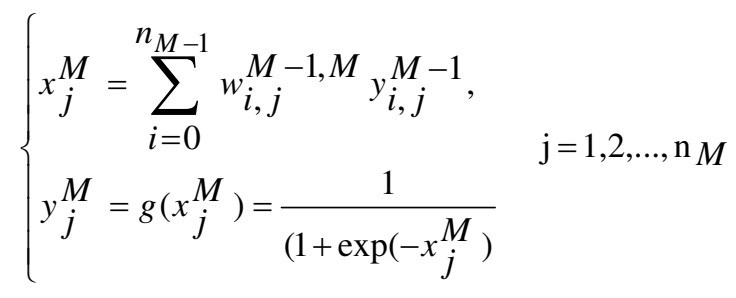

The weights $w_{i, j}^{l-1, l}$ of a neural network are changed during training to reduce the error function. The following is a commonly used training error function.

$$
E=\sum_{s=1}^{S} E_{S}=\frac{1}{2} \sum_{s=1}^{S} \sum_{i=1}^{N_{M}}\left(t_{i}^{S}-y_{i}^{s, M}\right)
$$

Where $y_{i}^{s, M}$ is the output of $i$ th neuron of $M$ th layer when inputting sth sample.

\subsubsection{Algorithm for Grasshopper Optimization (GOA)}

A population-based strategy, the grasshopper optimization algorithm. The natural behaviour and life cycle of grasshoppers can be used to solve optimization problems in this manner. The lack of exploration and exploitation balancing in meth-heuristics approaches is successfully overcome with this swarm intelligence optimization technique. Grasshoppers, like bees and ants, live in groups. The grasshoppers' community may be seen not only in adulthood, but also in the nymph stage, living in a collective style.A solitary grasshopper flies arbitrarily in the surrounding region in search of food; yet, once it joins the swarm, it goes incongruently to 
the community in a predetermined course. Grasshoppers in nature search for food in rolling cylinder pathways, where they destroy agricultural crops. The following is a mathematical representation of grasshopper swarming behaviour.

$$
x_{i}=S_{i}+G+A
$$

Where does the I grasshopper fit in,The wind direction is $A$, and $G$ represents the force of gravity pulling on the I grasshopper. In a group, $\mathrm{Si}$ is the symbol for social interaction. Expand $\mathrm{Si}, \mathrm{G}$, and $\mathrm{A}$ in the equation to get the following result: (eq 13).

$$
x_{i}=\sum_{j=1, j \neq 1}^{N} s\left(\left|x_{j}-x_{i}\right|\right) \frac{x_{j}-x_{i}}{d_{i j}}-g e_{g}+u e_{w}
$$

where $s(r)=f e^{-r / l}-e^{-r}$ is a function simulating the impact of social interactions and $\mathrm{N}$ is the number of grasshoppers. $g e_{g}$ is the expanded $\mathrm{G}$ component, where $\mathrm{g}$ is the gravitational force and $e_{g}$ is a unit vector pointing toward the center of the earth. $u e_{w}$ is the expanded A component, where $\mathrm{u}$ is a constant drift and $e_{W}$ is a unit vector pointing in the direction of the wind. $\mathrm{d}_{i j}$ is the distance between the $i$ and $j$ grasshoppers and is calculated as $d_{i j}=\left|x_{-} x_{i} j\right|$.

The influences of wind and gravity are significantly weaker than the relationships between grasshoppers, hence this mathematical model should be changed as follows:

$$
x_{i}=c\left(\sum_{j=1, j \neq 1}^{N} c \frac{u b-l b}{2} s\left(\left|x_{j}-x_{i}\right|\right) \frac{x_{j}-x_{i}}{d_{i j}}\right)+T_{d}
$$

Whereas $u b$ and $l b$ perform the upper and lower boundaries of the search space, $\mathrm{T}_{\mathrm{d}}$ is the optimal solution value, and $\mathrm{c}$ is a decreasing coefficient that balances the exploitation processes, as follows:

$$
c=c_{\max }-i t e r \frac{c_{\max }-c_{\min }}{\text { Max }_{\text {iter }}}
$$

Where $c_{\max }$ is the maximum value, $c_{\min }$ is the minimum value, iter presents the current iteration and Maxiter is the maximum number of iterations.

\section{Hybrid Grasshopper Optimization Algorithm}

The weight was estimated with the help of an ANN. Then, using the GOA, optimise the weight in order to choose the best path for transmitting data packets from source to destination without causing traffic. Using a function that is a measure of the optimal performance quality of the response provided, this algorithm can concurrently find the best network structure and weights. The following is a formula for the grasshopper optimization algorithm-based approach of selecting the best path for the weights:

Step 1: A randomly selected population is used as a starting point. A random set of ANN weights characterises each member of the first generation.

Step 2:Using the weights associated with each individual, the ANN was utilised to determine the outputs for all $\mathrm{S}$ experimental inputs. The predicted outputs were then compared to the experimental data once more.

Step 3:The lowest E (neural network error values Eq) values were used to rank the population (12).

Step 4:The person in the population with the lowest E value (the best person) was passed down to the following generation. 
Step5:Update the solution using the Grasshopper Algorithm after the fitness evaluation. We can revise the solution using equation (15). Mathematically, the grasshopper xi's circumstance or location can be described.

Step6. Reduce parameter $\mathrm{c}$ according to the iteration numbers to achieve a balance between exploration and exploitation. As the repetition progresses, this technique promotes an improvement in exploitation. Equation determines the coefficient $\mathrm{c}$. (16). Figure 6 illustrates the overall ANN-GOA flowchart.

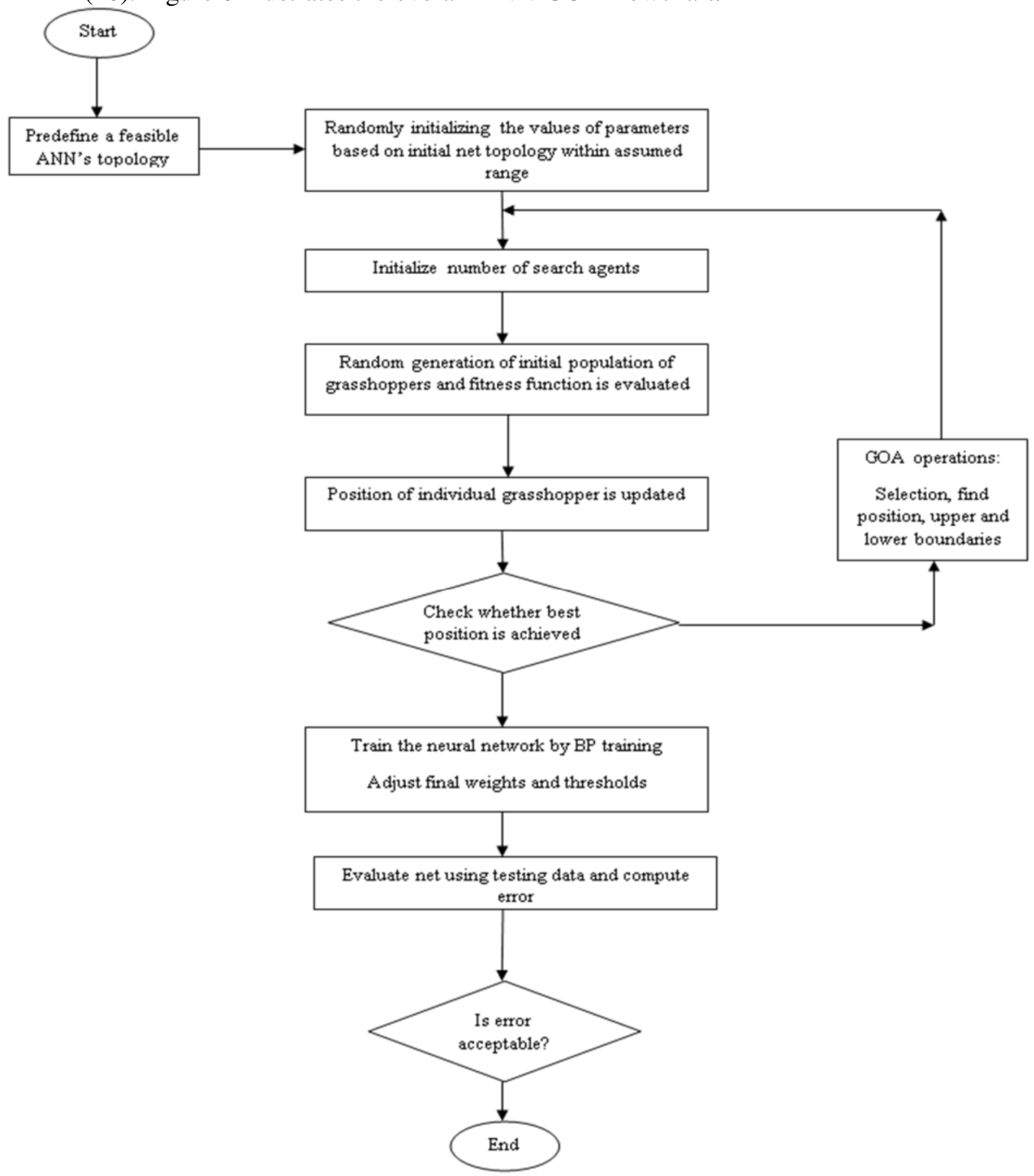

Figure 6: Flowchart of ANN-GOA

\section{Result and Discussion}


To simulate the specified protocol structure, the MATLAB software is utilised. Table 1 lists the simulation parameters. Around 200 sensor nodes are made and placed at random in a $1000 \mathrm{~m} \times 1000 \mathrm{~m}$ region, each one spanning $100 \mathrm{~m} 2$. In the initial stage of this work, the nodes are clustered using one hop and roughed using EANN-GOA. SEEP [21], EACRP [22], ME-CBCCP [23], and TDEEC [24] are then compared in terms of network throughput, endto-end delay, packet delivery ratio, network lifetime, energy consumption, and scalability.

Table1: Simulation parameters.

\begin{tabular}{|l|l|}
\hline Specification & Value \\
\hline Area for simulation & $1000 \times 1000 \mathrm{~m}$ \\
\hline Range of node coverage & $100 \mathrm{~m}$ \\
\hline ID of the node & 16 bit \\
\hline Size of a typical packet & 50 bytes \\
\hline Nodes in the graph & 200 \\
\hline Time for simulation & $500 \mathrm{~s}$ \\
\hline Time between messages & $0.02 \mathrm{~s}$ \\
\hline $\begin{array}{l}\text { Protocol for the MAC } \\
\text { layer }\end{array}$ & IEEE 802.15 .4 \\
\hline The energy of nodes & $100 \mathrm{~J}$ \\
\hline
\end{tabular}

\subsection{Network throughput}

It is characterized because the most quantity of bit may be transmitted effectively inside one scheduled time. The full output of the system may be characterized because the total of the output that's created by the effective direct transmissions additionally to the output determined by the cooperation section when inaccurate packet receptions. This is precisely drawn as,

$$
\begin{gathered}
N T_{\text {tot }}=N T_{D}+N T_{c o} \\
N T_{D}=\left(1-\overline{P C E_{S \rightarrow D}}\right) * \frac{N P_{\text {load }}}{\bar{T} N_{D}}(18) \\
N T_{c o}=2 * \overline{P C E_{S \rightarrow D}} * \frac{N P_{l d}}{\bar{T} N_{\text {tot }}}(19)
\end{gathered}
$$

In equation (17), the packet error rate is given by $\overline{P C E_{S \rightarrow D}}$, while $N P_{l d}$ signifies the normal packet payload. Additionally, $N T_{D}$ is the average time it takes for a single data packet to be transmitted and $\bar{T} N_{\text {tot }}$ refers to the total amount of time it takes to complete a cooperative relay transmission. In terms of throughput, the suggested protocol is compared to the existing SEEP, EACRP, ME-CBCCP, and TDEEC protocols in figure 7. 


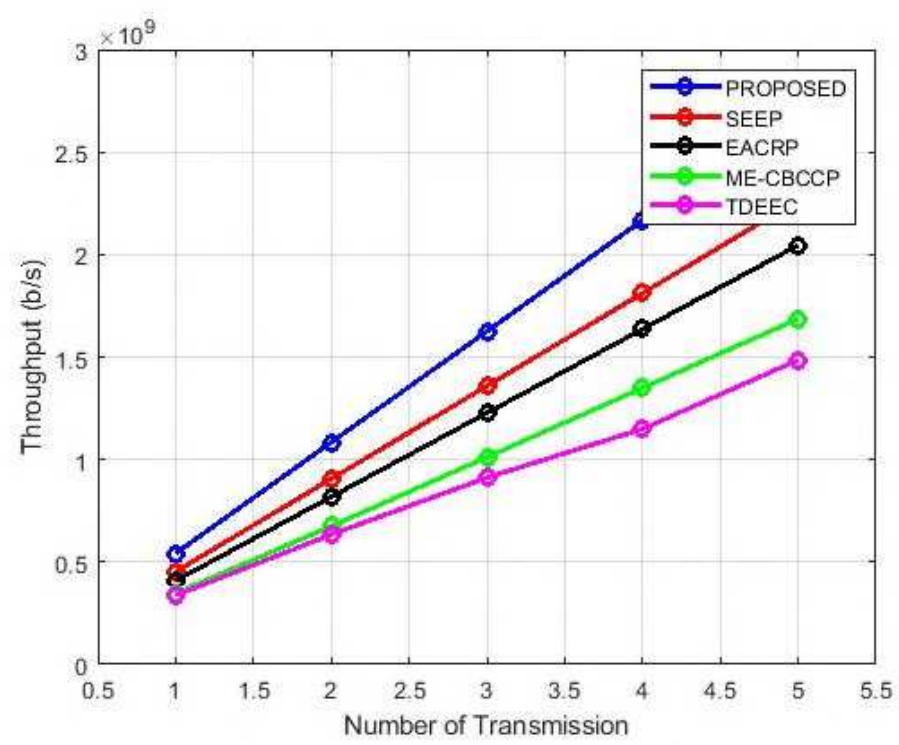

Figure7: Performance analysis of Network throughput

Figure 7illustrates that $N T_{\text {tot }}$ our proposedprotocol outperforms the existing SEEP, EACRP, ME-CBCCP, and TDEEC protocols by a significant margin. The proposed value is $5.32 \times 10^{8}(\mathrm{~b} / \mathrm{s})$, while SEEP is $4.33 \times 10^{8}(\mathrm{~b} / \mathrm{s})$, which is comparable to the EACRP's $4.06 \mathrm{X}$ $10^{8}(\mathrm{~b} / \mathrm{s})(\mathrm{b} / \mathrm{s})$. With $3.37 \times 10^{8}(\mathrm{~b} / \mathrm{s})$ and $1.15 \times 10^{8}(\mathrm{~b} / \mathrm{s})$, the ME-CBCCP and TDEEC achieve the wickedest value.

\subsection{End-to-end delay}

The $E N D_{t}$ is move on from the initial transmission till the proper reception within the destinations may be characterized as

$$
E N D_{t}=N T_{D}+N T_{c o}
$$

Where $N T_{c o}$ is cooperative transmission through relays.

$$
N T_{c o}=N T_{\text {min-delay }}+N T_{\text {additional }}
$$

Where, $N T_{\text {min-delay }}$ is the minimum average delay of schedule, $N T_{\text {additional }}$ is an additional delay. The evaluation graph of the proposed protocolwith existing SEEP, EACRP, ME$\mathrm{CBCCP}$, and TDEECprotocolsin regards of $E N D_{t}$ is depicted in figure 8.

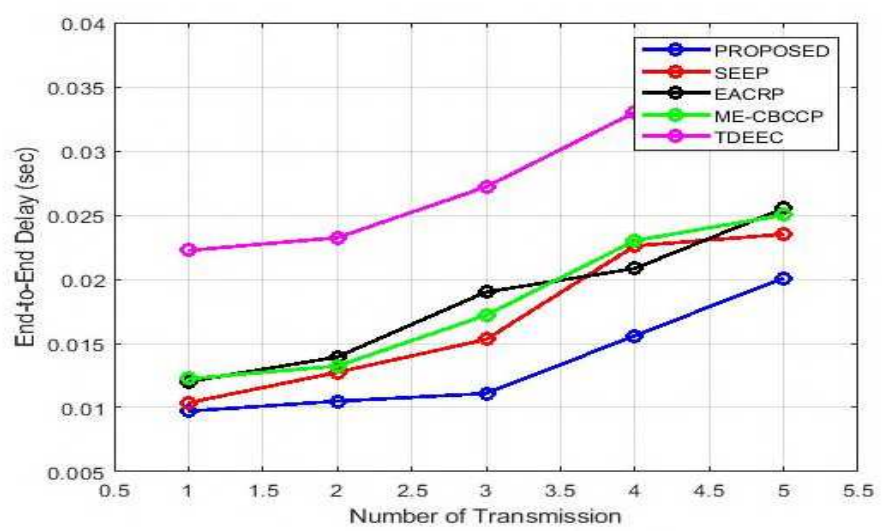

Figure 8: Performance analysis of End-to-end delay 
Figure 8 shows that $E N D_{t}$ our proposedprotocol $E N D_{t}$ is $9.72 \mathrm{X} 10^{-3}$ seconds which is expressively lesser than the existing SEEP, EACRP, ME-CBCCP, and TDEEC protocols. The $E N D_{t}$ value of the existing SEEPis 0.010 seconds,EACRP achieves 0.012 seconds. The ME-CBCCP and TDEEC result in the wickedest $E N D_{t}$ value with 0.012247 and 0.022257 seconds.

Our proposedprotocol $E N D_{t}$ takes $9.72 \times 10^{-3}$ seconds, which is significantly shorter time than the existing SEEP, EACRP, ME-CBCCP, and TDEEC protocols (see Figure 8). The $E N D_{t}$ current SEEP value is 0.010 seconds, whereas EACRP achieves 0.012 seconds. With 0.012247 and 0.022257 seconds, the ME-CBCCP and TDEEC produce the most terrible values.

\subsection{Packet delivery ratio}

The packet delivery ratio is the proportion of data packets received by destinations to those produced by sources. It's generally written as:

$$
\overline{P K D R}=\frac{\sum P K T_{\text {recvd }}}{\sum P K T_{\text {gen }}}
$$

Where, $\sum P K T_{\text {recvd }}$ is the totality of data packets received and $\sum P K T_{\text {gen }}$ is the totality of data packets generated.

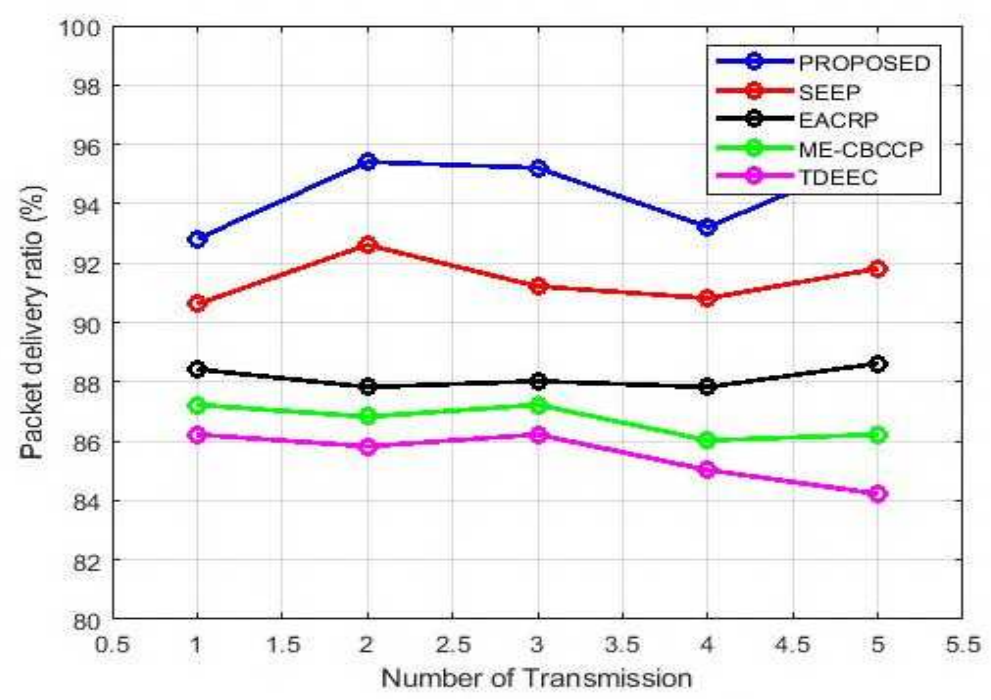

Figure 9: Performance analysis of Packet delivery ratio

Figure 9 shows that $\overline{P K D R}$ our proposed protocol is suggestively better than the existing SEEP, EACRP, ME-CBCCP, and TDEEC protocols. The $\overline{P K D R}$ of the proposed protocol is $95.81 \%$ and the existing SEEPis $92.62 \%$, EACRP88.62\%. The wickedest $\overline{P K D R} 87.22 \%$, $84 \%$ is achieved for existing ME-CBCCP, and TDEEC.

\subsection{Network lifetime}

The residual energy of network nodes at every scheduling vacancy are referred to as network lifespan. 


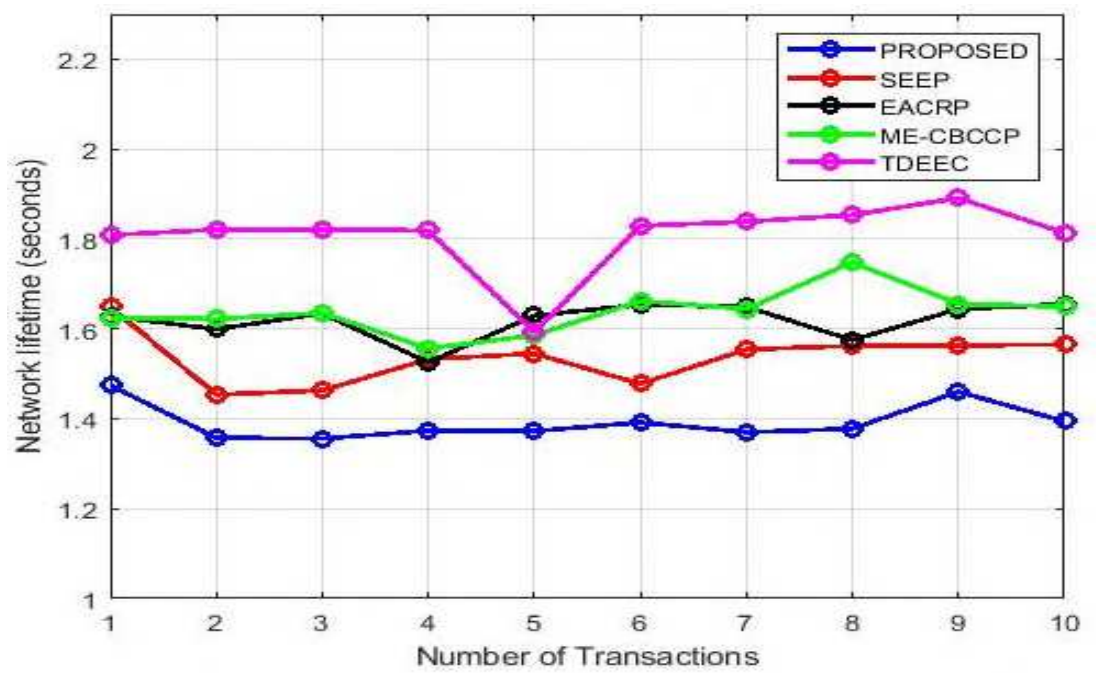

Figure 10: Performance analysis of Network lifetime

In figure 10 the network lifetime for the proposed protocol,and existing protocols SEEP, EACRP, ME-CBCCP, and TDEEC are plotted. Here the proposed protocolnetwork lifetime is small compare to the existing methods that are determined.

\subsection{Scalability}

Figure (11) presents the performance analysis of the scalability. Compared with existing methods our proposed method outcomes the highest scalability to transfer packets. Compared proposed protocol,with existing SEEP, EACRP, ME-CBCCP, and TDEECprotocol

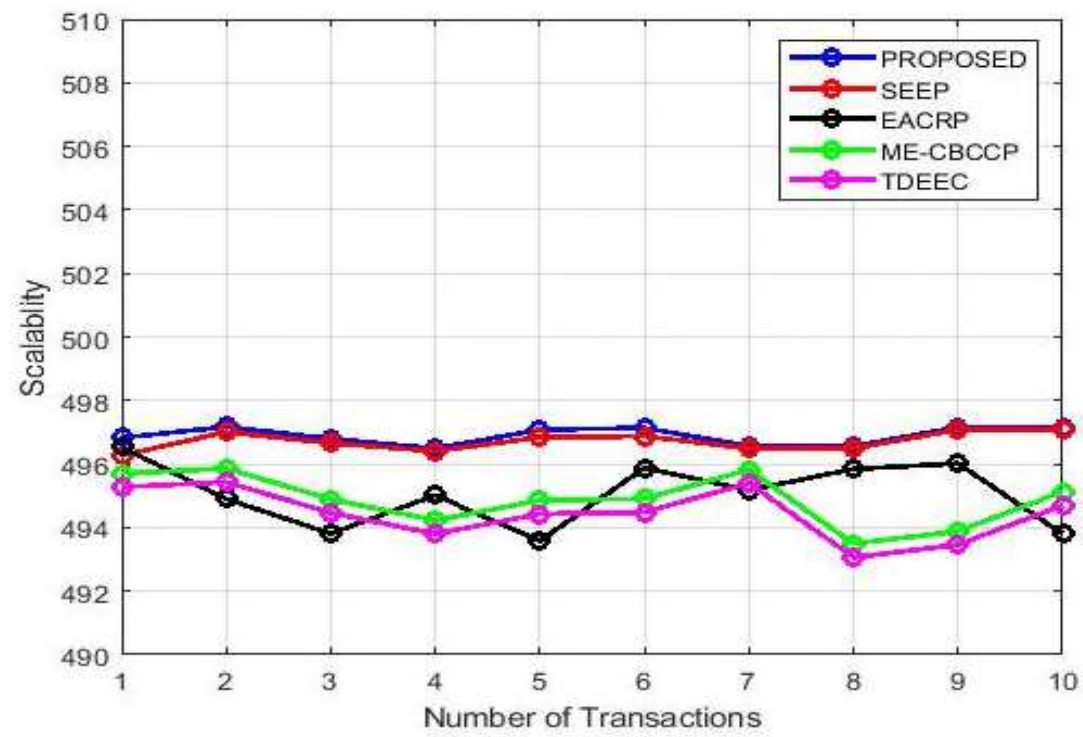

\subsection{Energy efficiency}

Figure 11: Performance analysis of Scalability

The energy consumption of three elementary energy efficiency potency procedures in demonstrating energy efficiency of each node as electronic communication, sensing, and information handling. Here the battery exhaustion procedure is spoken to as pursues

$$
E N_{i}(t)=E N_{i}-\sum_{k=1}^{t}\left(E N_{i}^{C}(j)+E N_{i}^{S}(j)+E N_{i}^{D}(j)\right)
$$

Where, $E N_{i}$ is the battery's underlying energy in a specific situation time 0 and $E N_{i}(t)$ is the rest of the energy in a given time $t$ for the smart thing $i . E N_{i}^{C}(j)$ is the shrewd thing's 
communication energy, which refers to the amount of packets communicated. $E N_{i}^{S}(j)$ $E N^{D}(j)$ Calculate the amount of energy used for each type of sensing and data processing.

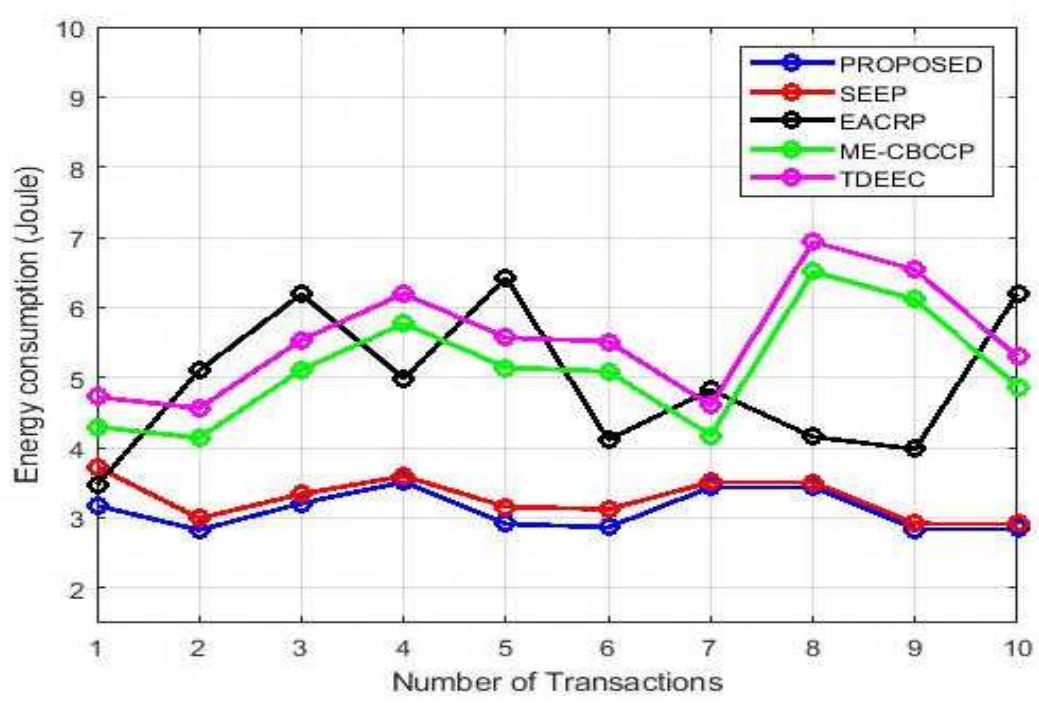

Figure 12: Performance analysis of Energy consumption

Figure (12) presents the performance analysis of the energy efficiency. Our proposed SSE protocol takes less energy to transmit the data packets.

\section{Conclusion}

The SSE routing protocol was defined in this paper, which is based on the Scalable, Secure, and Energy Efficient Routing protocol. It sends data packets from the source to the destination via the least congested path possible. Data from the cloud was initially acquired using a base station. The four parameters that go into picking a node (data packets) using a single-hop clustering approach are residual energy, distance to the sink, node density, and network average energy. The data packets are then encrypted with Fuzzy Mean Weighted Code (FMWC). The EANN-GOA method is then used to choose the least congested path from the source to the destination for data transfer. The proposed result is compared to a number of methods as well as some previous work. In comparison to previous research, our proposed methodology produces better results.

\section{Declarations}

Funding : In this research article has not been funded by anyone.

Conflict of interest : All authors do not have any conflict of interest.

Data availability : Not applicable.

Ethical Approval : This article does not contain any studies with human participants or animals performed by any of the authors.

\section{REFERENCES}

1) Pal, Raju, Subash Yadav, and Rishabh Karnwal. "EEWC: energy-efficient weighted clustering method based on genetic algorithm for HWSNs." Complex \& Intelligent Systems (2020): 1-10.

2) Rawat, Piyush, Siddhartha Chauhan, and Rahul Priyadarshi. "A Novel Heterogeneous Clustering Protocol for Lifetime Maximization of Wireless Sensor Network." Wireless Personal Communications (2020): 1-17. 
3) Manchanda, Rachit, and Kanika Sharma. "Energy efficient compression sensingbased clustering framework for IoT-based heterogeneous WSN." Telecommunication Systems (2020): 1-20.

4) Shukla, Anurag, and Sarsij Tripathi. "A multi-tier based clustering framework for scalable and energy efficient WSN-assisted IoT network." Wireless Networks (2020): $1-23$.

5) Mittal, Nitin, Simrandeep Singh, Urvinder Singh, and Rohit Salgotra. "Trust-aware energy-efficient stable clustering approach using fuzzy type-2 Cuckoo search optimization algorithm for wireless sensor networks." Wireless Networks 27, no. 1 (2021): 151-174.

6) Baradaran, Amir Abbas, and Keivan Navi. "HQCA-WSN: High-quality clustering algorithm and optimal cluster head selection using fuzzy logic in wireless sensor networks." Fuzzy Sets and Systems 389 (2020): 114-144.

7) Salam, Taspia, and Md Sharif Hossen. "Performance Analysis on Homogeneous LEACH and EAMMH Protocols in Wireless Sensor Network." Wireless Personal Communications (2020): 1-34.

8) Sharma, Neelam, B. M. Singh, and Karan Singh. "QoS-based energy-efficient protocols for wireless sensor network." Sustainable Computing: Informatics and Systems 30 (2021): 100425.

9) Poonam, Rani, and Sharma Avinash. "Minimum Spanning Tree Structure Based Routing Technique for Homogeneous Wireless Sensor Network." Journal of Computational and Theoretical Nanoscience 17, no. 6 (2020): 2763-2767.

10) Alghamdi, Turki Ali. "Energy efficient protocol in wireless sensor network: optimized cluster head selection model." Telecommunication Systems (2020): 1-15.

11) Ansari, Abdul Rahim, and Sunghyun Cho. "CHESS-PC: cluster-HEad selection scheme with power control for public safety networks." IEEE Access 6 (2018): 51640-51646.

12) Osamy, Walid, Ahmed Salim, and Ahmed M. Khedr. "An information entropy based-clustering algorithm for heterogeneous wireless sensor networks." Wireless Networks 26, no. 3 (2020): 1869-1886.

13) Kavitha, A., Koppala Guravaiah, and R. Leela Velusamy. "A cluster-based routing strategy using gravitational search algorithm for wsn." Journal of Computing Science and Engineering 14, no. 1 (2020): 26-39.

14) Mervat Mustafa Raouf "Clustering in Wireless Sensor Networks (WSNs)"2019

15) Li, Na, Arnaud Martin, and Rémi Estival. "Heterogeneous information fusion: Combination of multiple supervised and unsupervised classification methods based on belief functions." Information Sciences 544 (2021): 238-265.

16) Sharma, Sukhwinder, Rakesh Kumar Bansal, and Savina Bansal. "Heterogeneityaware Energy-efficient Clustering (HEC) Technique for WSNs." TIIS 11, no. 4 (2017): 1866-1888.

17) Aimtongkham, Phet, Sovannarith Heng, Paramate Horkaew, Tri Gia Nguyen, and Chakchai So-In. "Fuzzy logic rate adjustment controls using a circuit breaker for persistent congestion in wireless sensor networks." Wireless Networks 26, no. 5 (2020): 3603-3627.

18) Muzzammil, Muhammad, Niaz Ahmed, Gang Qiao, Imran Ullah, and Lei Wan. "Fundamentals and Advancements of Magnetic-Field Communication for Underwater Wireless Sensor Networks." IEEE Transactions on Antennas and Propagation 68, no. 11 (2020): 7555-7570. 
19) Wang, Quan, Deyu Lin, Pengfei Yang, and Zhiqiang Zhang. "An energy-efficient compressive sensing-based clustering routing protocol for WSNs." IEEE Sensors Journal 19, no. 10 (2019): 3950-3960.

20) Thangaramya, K., Kanagasabai Kulothungan, R. Logambigai, M. Selvi, Sannasi Ganapathy, and Arputharaj Kannan. "Energy aware cluster and neuro-fuzzy based routing algorithm for wireless sensor networks in IoT." Computer Networks 151 (2019): 211-223.

21) Shukla, Anurag, and Sarsij Tripathi. "A multi-tier based clustering framework for scalable and energy efficient WSN-assisted IoT network." Wireless Networks (2020): 1-23.

22) Darabkh, K. A., Al-Maaitah, N. J., Jafar, I. F., \& Ala'F, K. (2018). EA-CRP: A novel energy-aware clustering and routing protocol in wireless sensor networks. Computers \& Electrical Engineering, 72, 702-718.

23) Rani, S., Talwar, R., Malhotra, J., Ahmed, S. H., Sarkar, M., \& Song, H. (2015). A novel scheme for an energy efficient Internet of Things based on wireless sensor networks. Sensors, 15(11), 28603-28626.

24) Saini, P., \& Sharma, A. K. (2010). Energy efficient scheme for clustering protocol prolonging the lifetime of heterogeneous wireless sensor networks. International Journal of computer applications, 6(2), 30-36.

25) Rani, Poonam, and Avinash Sharma. "Linear Scalable Routing Protocol for Wireless Sensor Network." In IOP Conference Series: Materials Science and Engineering, vol. 1057, no. 1, p. 012094. IOP Publishing, 2021.

26) Poonam, Rani, and Sharma Avinash. "Minimum Spanning Tree Structure Based Routing Technique for Homogeneous Wireless Sensor Network." Journal of Computational and Theoretical Nanoscience 17, no. 6 (2020): 2763-2767.

27) Poonam, Rani, and Sharma Avinash. "Cluster Based Data Aggregation Scheme in Wireless Sensor Network." Journal of Computational and Theoretical Nanoscience 17, no. 6 (2020): 2678-2683. 